\title{
Spatial Knowledge Acquisition During MR-based Navigation in Complicated Environments
}

\author{
Bing Liu ${ }^{\mathrm{a}}$ *, Shengkai Wang ${ }^{\mathrm{a}}$ \\ ${ }^{a}$ Chair of Cartography, Technical University of Munich, Germany - bing.l@tum.de, shengkai.wang@tum.de \\ * Corresponding author
}

Keywords: Mixed Reality, Spatial Knowledge Acquisition, Navigation, Visual Attention

\begin{abstract}
:
Cartography and location based services benefits a lot from the booster of extended reality, which includes augmented reality, mixed reality and virtual reality. Among these technologies, mixed reality (MR) is widely used in navigation. MR is new experience to most users and thus it is usually the virtual holograms that attracts the users. However, during navigation, user need to be aware of the physical world to stay safe. Besides, similar with smartphone-based navigation, we cannot afford the complete loss of spatial knowledge. Therefore, many studies are trying to include spatial knowledge acquisition during MR-based navigation.

It has been proved that spatial knowledge acquisition can be kept without much workload, e.g. Liu and colleagues (2021) confirmed that virtual semantic landmarks used in simple and quiet built areas can assist the corresponding information learning (Figure 1). However, it remains a question, how users respond to such landmarks in much busier and more complicated environment.
\end{abstract}

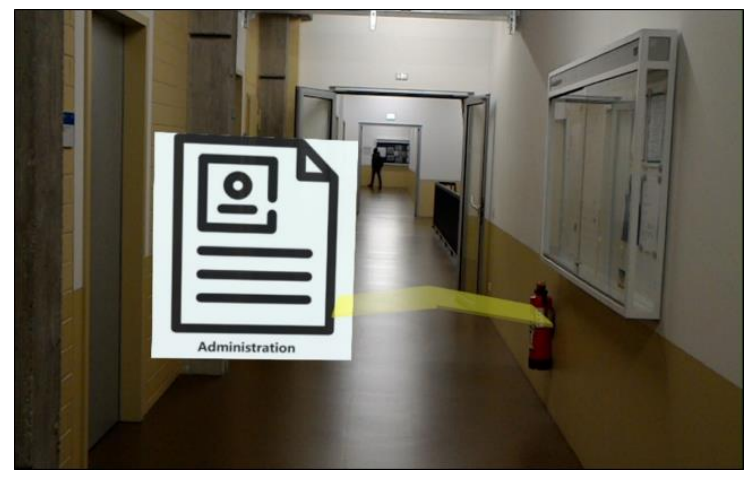

Figure 1. Virtual semantic landmark in simple and quiet environment (Liu et. al. 2021).

A busy and complicated area is different from the aforementioned simple and quiet one. In such areas, it requires a lot more visual attention to the physical world. First, it's complicated per se and the user needs to intentionally "pick up" the important objects. Besides, they need to stay alert to the upcoming events, e.g. in a supermarket, the consumer needs to pay attention to the passers-by, especially children and toddlers. In such situations, it's not clear yet if the virtual landmarks can still pop out.

Therefore, in this ongoing work, we propose to filter the irrelevant or less important information in such areas. We aim to reduce user's workload of processing the physical world, arrange the holograms based on view management theories and spare them more attention to the holograms and get more spatial knowledge as designed by the navigation provider. Two research questions are to be answered:

a) how to identify which information is less important;

b) if certain information is filtered out, will the users feel unsafe or less confident navigating in the areas. 